\title{
Mid-infrared Magnetic Mirror Based on a Hybrid Metal/Dielectric Metasurface
}

\author{
Ming Ye ${ }^{1}$, Shiqiang Li $^{1}$, Yang Gao ${ }^{1}$, Vivek Raj Shrestha ${ }^{2}$, and Kenneth B. Crozier ${ }^{* 1,2}$ \\ ${ }^{1}$ Department of Electrical and Electronic Engineering, University of Melbourne, Victoria 3010, Australia \\ ${ }^{2}$ School of Physics, University of Melbourne, Victoria 3010, Australia \\ *-Email: kenneth.crozier@unimelb.edu.au
}

\begin{abstract}
We propose a hybrid metal/dielectric metasurface that functions as a mid-infrared magnetic mirror. It consists amorphous silicon cuboids on gold. The physical mechanism is explained by image theory. Measured reflection spectra agree with simulations. (C) 2018 The Author(s) OCIS codes: (350.4238) Nanophotonics and photonic crystals; (160.3918) Metamaterials
\end{abstract}

\section{Introduction}

Metasurfaces enable versatile control over the wave-front of light upon reflection and refraction [1]. The magnetic mirror has drawn considerable interest, as it reflects with zero phase shift of the electric field. The electric field on the mirror surface is thus large (anti-node of standing wave), and an ultra-thin material placed on it can absorb strongly. Magnetic mirrors have been demonstrated comprising metal-insulator-metal (MIM) structures, but have low reflectance [2-3]. Magnetic mirrors based on magnetic dipole resonances in dielectric nanoparticles have been demonstrated [4]. Here, we propose a mid-infrared magnetic mirror based on a hybrid metal/dielectric metasurface. Our device comprises an amorphous silicon (a-Si) cuboids on gold. Compared to magnetic mirrors based on dielectric nanoparticles, our device is thinner and has high reflectance at wavelengths away from the resonance. We begin by showing that a high index cuboid sitting on a perfect electric conductor (PEC) can support a magnetic dipole resonance of the same nature to that of a high index cube that is of double the thickness (without PEC). We show through simulations that our structure offers magnetic mirror functionality across the range $\lambda=6-12 \mu \mathrm{m}$. We fabricate three designs. Measured reflection spectra are in good agreement with simulations.

\section{Results and discussions}

We begin by considering the role of the PEC using the method of images. This states that for a static charge distribution above a PEC, the potential above the PEC will be unchanged if one replaces the PEC with a charge distribution that forms a mirror image of the original charge distribution, but with the charges reversed in sign. We apply this to interpret scattering from an a-Si cube and from an a-Si cuboid sitting on a PEC. Normally-incident light of the appropriate wavelength can induce circling electric dipoles inside the a-Si cube forming a magnetic dipole (upper half of Fig. 1(a,i)). If we cut the cube in half and place it on a PEC (Fig. 1(a.ii)), the field pattern above the PEC should be of the same character. We can interpret the PEC as being replaced by image electric dipoles that mirror the original electric dipoles in the upper half of the cube (red arrows, Fig. 1(a.ii)). In the lower half of Fig. 1(a) we present simulations of the instantaneous electric fields for a single a-Si cube and for a single a-Si cuboid on PEC $(\lambda=$ $8.6 \mu \mathrm{m}$ ), demonstrating the excitation of magnetic dipole resonances. Perfectly matched layers are used at the boundaries of the simulation domain. We plot the scattering cross sections (Fig. 1(b)), obtained by integrating the Poynting vector of the scattered field (=total field -incident plane wave). For the cuboid-on-PEC, the integration is over the outer boundary of the upper half space of the simulation domain. Both curves show a peak at $\lambda \sim 8.6 \mu \mathrm{m}$, identified as a magnetic dipole resonance. We next investigate our structure as a magnetic mirror via finite difference time domain (FDTD) simulation of the amplitude and phase of the reflected wave as a function of the cuboid side length $(L$, Fig. 1(c)). The a-Si thickness is $1 \mu \mathrm{m}$, and the gold is $100 \mathrm{~nm}$ thick. The period is $2 L$. The top surface of cuboids is chosen as the reference plane for phase shift calculation. The results are plotted as Fig. 1(d)-(e). It can be seen that as we increase the cuboid side length $L$, the major reflection dip (white dashed line, Fig. 1(d)), which is associated with the magnetic dipole resonance, shifts to longer wavelengths. The wavelength of zero phase shift (white dashed line, Fig. 1(e), magnetic mirror functionality), is similarly tuned by modifying $L$. It should be noted that the wavelength of zero phase shift tracks the reflection dip wavelength, but is not identical to it. Indeed, the reflection at zero phase shift wavelength exceeds 55\% over the spectral range $6-12 \mu \mathrm{m}$. Additional reflection dips that appear for large $L$ are attributed to higher order multipole resonances.

We next describe experimental results. Fabrication starts with e-beam evaporation of gold $(100 \mathrm{~nm})$ and a-Si onto a silicon wafer. Devices containing cuboids with side lengths of $L=(2.2 \mu \mathrm{m}, 2.6 \mu \mathrm{m}, 3.25 \mu \mathrm{m})$ are then formed 
by e-beam lithography and dry etching. For all three samples, the a-Si cuboids are $570 \mathrm{~nm}$ thick. Fig. 2(a) shows the SEM image of the fabricated structure, with zoom-in image of a single cuboid (Fig. 2(b)). Reflectance spectra are measured using a homebuilt microscope attached to a Fourier transform infrared (FTIR) spectrometer. It can be seen that measured and simulated reflection spectra (Fig. 2(c)) are in agreement. The small dips appearing at shorter wavelengths might be due to the illumination not being purely at normal incidence. Fig. 2(d) is the simulated phase shift. Zero phase shift occurs at $\lambda=6.76 \mu \mathrm{m}$ and $\lambda=7.18 \mu \mathrm{m}$ for the devices with $L=2.2 \mu \mathrm{m}$ and $L=2.6 \mu \mathrm{m}$, respectively. The slightly redshift of the zero phase shift points with respect to their corresponding reflection dips are due to our choice of reference plane being on the top surface of cuboid instead of the center. We further verify the magnetic mirror behavior of our structure by comparing the simulated standing wave pattern for light reflected from our structure and from a gold mirror (Fig. 2(e)-(f)). At magnetic dipole resonance, maximum electric field occurs on the cuboid surface. By contrast, it can be seen that for a gold mirror, the electric field exhibits a minimum at the goldair interface.

(a)

(i)
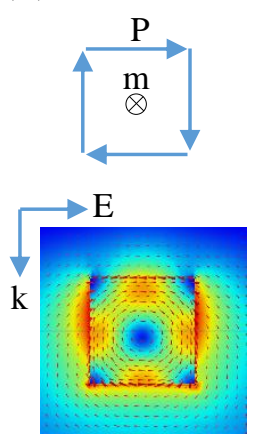

(ii)

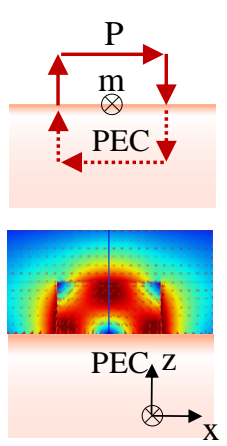

(b)

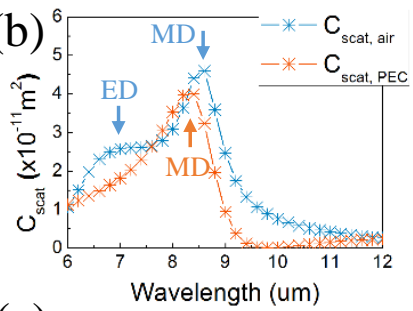

(c)

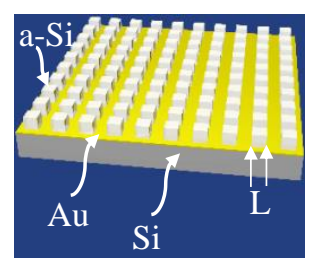

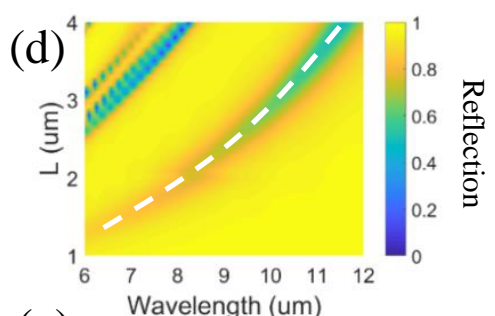

$(\mathrm{e})_{4}$

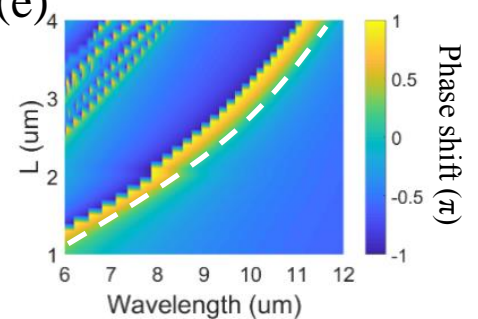

Fig. 1. (a) Magnetic dipole resonances excited by normally incident light (x-polarized) onto (i) a-Si cube ( $L=2 \mu m)(i i)$ a-Si cuboid $(L=2 \mu m$, thickness $=1 \mu \mathrm{m}$ ) on PEC. (b) Simulated scattering cross section for a-Si cube and a-Si cuboid on PEC described in (a). (c) Schematic of proposed magnetic mirror. $(\mathrm{d}, \mathrm{e})$ Simulated reflection and phase shift of the proposed structure (cuboid thickness $=1 \mu \mathrm{m}$ ).
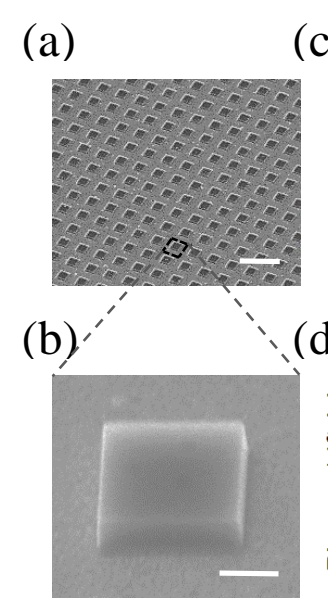

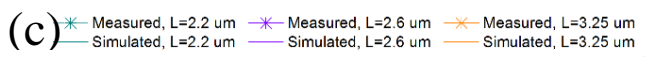
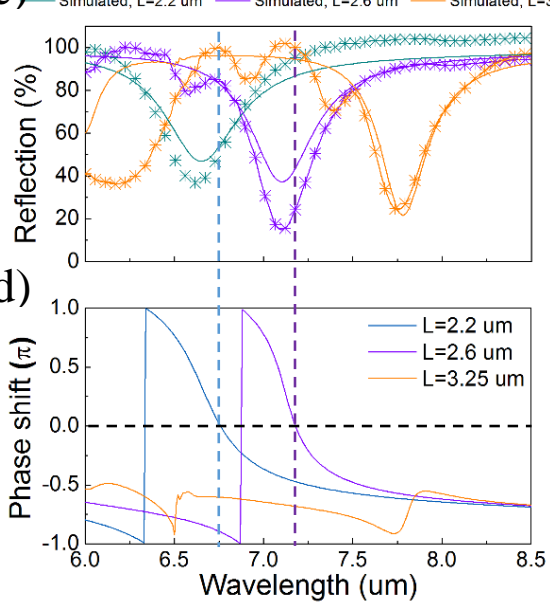

(e)

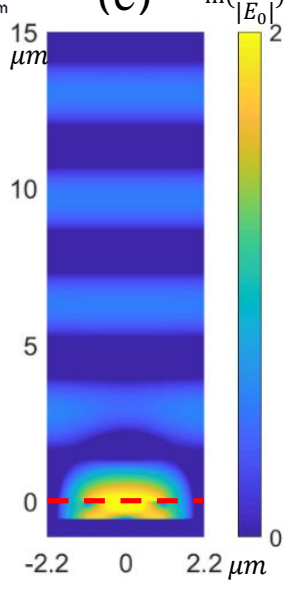

(f)

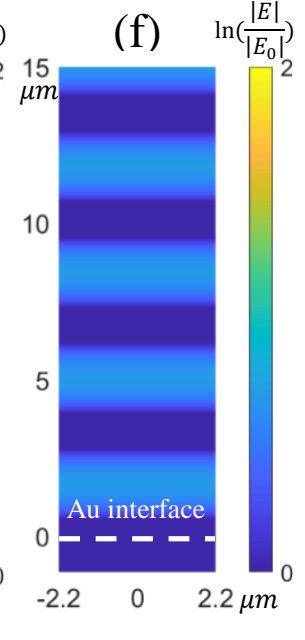

Fig. 2. (a,b) SEM images of fabricated structure. Scale bars are $8 \mu m$ (upper) and $1 \mu m$ (lower). (c) Measured and simulated reflection spectra of fabricated structures. (d) Simulated phase shift. Simulated standing wave pattern for (e) proposed structure $(L=2.2 \mu \mathrm{m})$ and (f) gold mirror, both at $\lambda=6.76 \mu \mathrm{m}$. Dashed line indicates reference plane.

\section{References}

[1] P. Genevet et al, "Recent advances in planar optics: from plasmonic to dielectric metasurfaces," Optica 4, 139-152 (2017).

[2] M. Sherrott et al, "Experimental Demonstration of $>230^{\circ}$ Phase Modulation in Gate-Tunable Graphene-Gold Reconfigurable Mid-Infrared Metasurfaces." Nano Letters 17, 3027-3034 (2017).

[3] J. Park et al. "Dynamic reflection phase and polarization control in metasurfaces." Nano letters 17, 407-413 (2016)

[4] S. Liu et al, "Optical magnetic mirrors without metals," Optica 1, 250-256 (2014) 


\section{University Library}

\section{- M M N E R VA A gateway to Melbourne's research publications}

Minerva Access is the Institutional Repository of The University of Melbourne

Author/s:

Ye, M;Li, S;Gao, Y;Shrestha, VR;Crozier, KB

Title:

Mid-infrared Magnetic Mirror Based on a Hybrid Metal/Dielectric Metasurface

Date:

2018-01-01

Citation:

Ye, M., Li, S., Gao, Y., Shrestha, V. R. \& Crozier, K. B. (2018). Mid-infrared Magnetic Mirror Based on a Hybrid Metal/Dielectric Metasurface. 2018 CONFERENCE ON LASERS AND ELECTRO-OPTICS (CLEO), Part F93-CLEO_QELS 2018, IEEE. https://doi.org/10.1364/ CLEO_QELS.2018.FTh4J.6.

Persistent Link:

http://hdl.handle.net/11343/294890 